\title{
¿Por qué deben estudiar matemática los gobernantes? Paideia, dialéctica y política
}

\author{
Why do the rulers have to study philosophy? \\ Paideia, Dialectics and Politics
}

HENAR LANZA GONZÁLEZ ${ }^{\mathrm{a}}$

\section{Resumen}

Debido a que no es evidente en qué sentido el estudio de la matemática ayuda a los guardianes de la República de Platón a dominar las pasiones y los prepara para ser gobernantes, mostraré la función propedéutica de la matemática; cómo las cinco disciplinas en las que está dividida - aritmética, geometría, estereotomía, astronomía y música - pueden dividirse a su vez en dos: la popular y la filosófica, y es solo esta última la que provoca el giro del alma (пعрıүүүү่) hacia lo que es siempre. Esta es la razón por la cual se afirma que la matemática es una preparación para la dialéctica, el único método propiamente filosófico y la ciencia máxima. Solo tras una década de ejercitación matemática el guardián se convertirá en dialéctico, ascenderá al ámbito inteligible y contemplará las Ideas, divinas y ordenadas. Esto despertará en él el deseo de querer asemejarse a ellas y, por mímesis, volverse él también divino y ordenado (en la medida en la que esto le es posible al hombre), lo que le permitirá ejercer el gobierno de sí (દ่үкра́твıа), que en la psicología platónica se traduce en el mandato de lo racional sobre lo irascible y lo apetitivo, el gobierno de lo intelectual sobre lo anímico. A pesar del deseo de permanecer para siempre en el ámbito inteligible, el dialéctico deberá descender nuevamente a la caverna y gobernar de forma justa la pólis e instaurar en las costumbres públicas y privadas de los gobernados el orden contemplado, alejado de la tiranía (el más degenerado de los regímenes políticos) y de las pasiones violentas y los apetitos que tiranizan al propio tirano, según el paralelismo alma - ciudad. Cierro con la respuesta a la pregunta de quién es ese filósofo-gobernante en el siglo XXI.

Palabras clave: Matemática. Paideia. Propedéutica. Dialéctica. Inconmensurables.

aUniversidad del Norte, Barranquilla, Atlántico, Colombia. Doctora en Filosofía, e-mail: lanzam@uninorte.edu.co 


\begin{abstract}
Because it is not evident in what sense Mathematical studies helps the guardians of Plato's Republic to dominate the passions and turn them into potential rulers, I show the propaedeutic function of Mathematics; how the five disciplines in which it is divided - Arithmetic, Geometry, Stereotomy, Astronomy and Music - can be divided into two: popular and philosophical, and it is only the latter that causes the

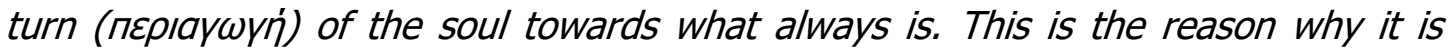
stated that Mathematics is a preparation for Dialectics, the one and only properly philosophical method and the maximum Science. Only after one decade of mathematical exercise will the guardian become a dialectician, ascend to the intelligible realm and contemplate Ideas, divine and ordered. It will be then when he will want to resemble them and become divine as well and ordered to the extent that this is possible for a man, what will allow him to practice the self-government

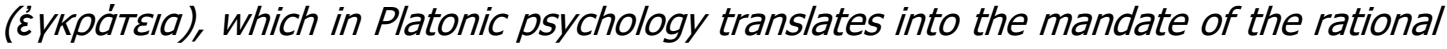
over the irascible and the appetitive, the government of the intellectual over the emotional. Despite the desire to remain forever in the intelligible realm, the dialectician must descend again to the cave to rule with justice the pólis and to establish in the public and private customs of the governed the order contemplated, away from tyranny (the most degenerate of the politicians regimes) and the violent passions and appetites that tyrannize the tyrant himself, according to the parallelism city-soul. I close with the answer to the question of who is that philosopher-ruler in the 21st century.
\end{abstract}

Keywords: Mathematics. Paideia. Propaedeutic. Immeasurable. Dialectics.

\title{
Introducción
}

No es Platón un autor que muestre impaciencia por lograr su fin de forma directa o apresurada, sino que son varios los diálogos en los que su escritura evidencia un gusto por preparar el terreno para alcanzar indirectamente su fin: para responder a la petición de Sócrates, Timeo retrotrae su relato hasta el estado precósmico del universo (Ti., 28c y ss.) y para conseguir que los ciudadanos cumplan la ley, se recurre a la persuasión por medio del preámbulo ( $\pi \rho \rho$ que se antepone a la ley misma ( $L g, 718 b-c$ y 722d). Análogamente, cuando está pensando cómo hacer que los mejores de los guardianes se conviertan en gobernantes justos, antepone una larga formación previa, conocida como "el curriculum matemático", (R., VII). 
Lo que mostraré es en qué sentido dicha formación matemática es propedéutica $^{1}$, cómo la ejercitación en aritmética, geometría (BADIOU, 2007; DE LORENZO, 1988; REY PASTOR y BABINI, 1997; SOLANA DUESO, 2008; VLASTOS, 1988; WEDBERG, 1955), estereonomía (ESCOBAR MONCADA, 1995), astronomía (ELENA y ORDÓÑEZ, 1988; LISI, 1991) y música (BARKER, 1994; GARCÍA CASTILLO, 1997) predispone al filósofo para la dialéctica (BROCHARD, 2008; KLEIN, 1992; MONTSERRAT, 1995; NOVAK, 1982 y 1983; VEGETTI, 2012; VLASTOS, 1988), la ciencia máxima que le permitirá aprehender las Ideas y estar en condiciones de gobernar de forma racional, alejado de la tiranía - el más degenerado de los regímenes políticos $(R$, VIII-IX) - y las pasiones violentas y los apetitos que tiranizan al propio tirano, según el paralelismo entre psyché y pólis (R., IV) (CANO CUENCA, 2018; LISI, 2018). En definitiva, responderé a la pregunta de por qué el futuro gobernante tiene que dar ese rodeo y estudiar matemática, (teniendo siempre presente que cuando Platón habla de matemática, se refiere a algo más amplio que hoy en día, mientras que cuando habla de razón usa el término en un sentido mucho más especializado (CAJORI, 1920, 57). Para ello, y siguiendo la propuesta de Phdr., 265e, dividiré el cuerpo matemático según sus articulaciones naturales para explicitar de qué modo contribuye cada una de las cinco disciplinas al ascenso hacia el ámbito inteligible. A continuación, aclararé la diferencia entre los métodos matemático y dialéctico (BADIOU, 2007 y 2014; CAJORI, 1920; DE LORENZO, 1974; KLEIN, 1992; REY, 1961), pero también cómo pudo haber influido el estudio de los números inconmensurables en la concepción platónica de la dialéctica (NOVAK, 1982 y 1983). Finalmente, con un movimiento descendente, el dialéctico regresa de nuevo a la caverna y gobierna la pólis (EGGERS LAN, 2000; LISI, 2018; VEGETTI, 2012), una anábasis y una catábasis que establecen una analogía entre el filósofo y el héroe.

\footnotetext{
1 про- "antes" у паıठєUтıко́s, "relativo a la enseñanza". https://stephanus.tlg.uci.edu/lsj/\#login=true
} 


\section{La pregunta de Platón}

Después de exponer cómo debería ser el mejor de los regímenes políticos, Platón se plantea "por medio de qué estudios" ( $\mu \alpha \theta \eta ́ \mu \alpha \tau \alpha, R ., 502 c)$ se formará a los filósofos, los únicos capaces de llevar a buen término el régimen descrito, cómo formar a los hombres que han de salir de la caverna y ascender desde las sombras hacia la luz del sol, qué será lo que propicie ese giro ( $\pi \varepsilon \rho ı \gamma \omega \gamma \eta ́)$ del

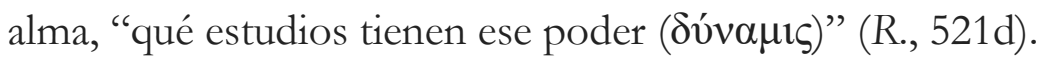

En adelante, Platón recurrirá en numerosas ocasiones a expresiones como ascenso hacia "la realidad" ( $\tau$ ò őv). ¿Por qué? Porque "el filósofo, para

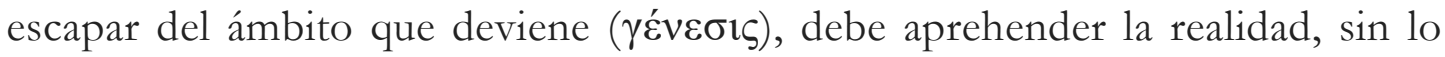

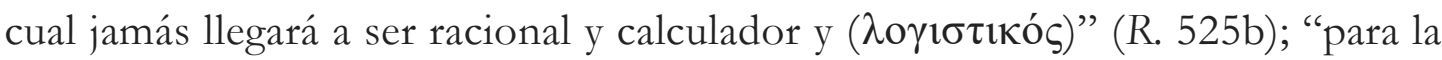

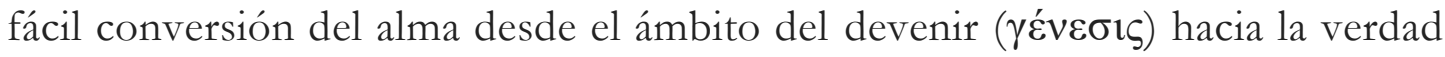
y la realidad (ov̉oía)" (R., 525c); "este estudio ha de resultarnos forzoso, puesto que obliga al alma a servirse de la inteligencia (vónoıs) para [alcanzar] la

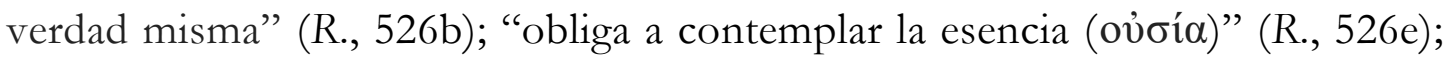

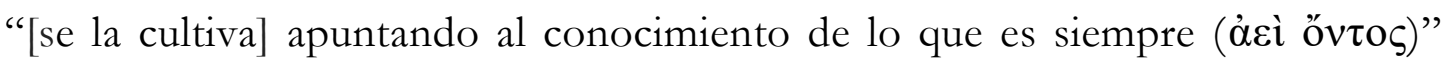
(R., 527b); que "atrae al alma hacia la verdad y que causa que el pensamiento

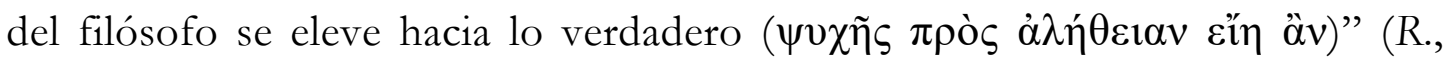
527b); estos estudios hacen que el órgano del alma "se purifique y resucite

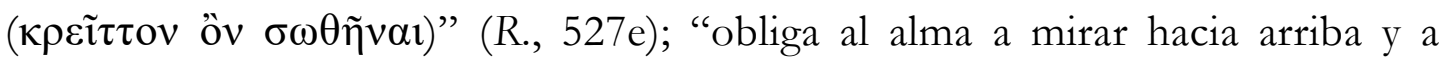

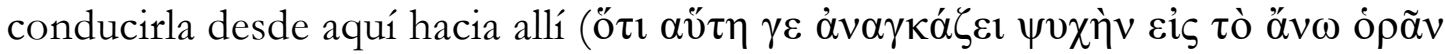

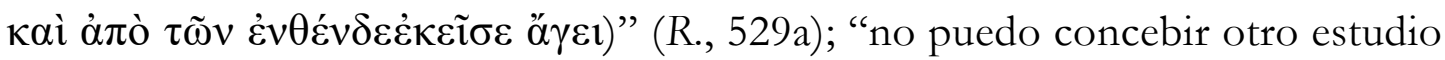
que haga que el alma mire hacia arriba que aquel que trata con el ser ( $\tau$ ò ôv) y con lo invisible" (R., 529b). El órgano del alma racional está como muerto, dormido, y el ascenso matemático ejerce sobre el filósofo un poder catártico que, tras liberarlo de aquello que lo adormece y le impide ver, lo activa.

La educación no consiste en introducir conocimiento en el alma, sino en volverla de la forma más rápida y eficaz hacia el mundo inteligible y el Bien, arrancándola del universo sensible [...] La paideia platónica no forma, sino descubre, quita el velo con el que los discursos falsos han cubierto el alma (LISI, 2018, p. 236). 


\title{
El camino de ascenso: la salida de la caverna. Matemática
}

\begin{abstract}
Platón sabe perfectamente que la racionalidad matemática es completamente obligatoria. Usted entra en la matemática y entonces tiene el ejemplo de una idealidad necesaria. Platón sabe muy bien que los argumentos filosóficos no tienen nunca la fuerza demostrativa de los argumentos matemáticos. [...] Una vez que entramos en la matemática estamos en la necesidad (BADIOU, 2007, p. 61, 62-63).
\end{abstract}

\section{Aritmética}

El pensamiento filosófico griego distinguió entre los conceptos abstractos y las cosas a las cuales estos son aplicados. Aristóteles definió el doble sentido de "número": número es lo numerado, lo numerable y aquello mediante lo cual numeramos" (Ph., IV 219b 5-7). Y: "Lo numerable es numerado por algo que es congénere, como por ejemplo las unidades por una unidad, los caballos por un caballo" (Ph., IV 223b 13-15).

Según Platón, la aritmética debe ser aprendida porque es el estudio común a todas las artes, ciencias y operaciones intelectuales, porque se ocupa del número y del cálculo y permite introducir orden en las enumeraciones ( $R$., 522c). La aritmética conduce hacia la verdad, hacia la contemplación de lo que es, porque

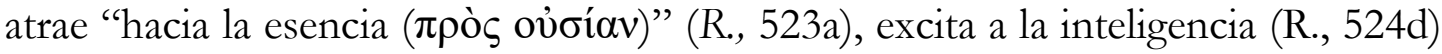

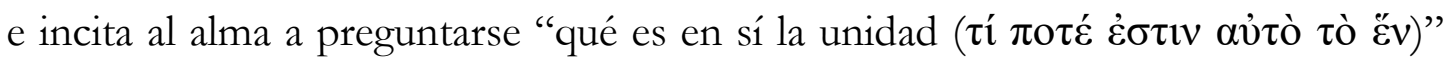
(R. 524e). Esta es la pregunta que debe ser capaz de responder el filósofo si quiere escapar del ámbito de la génesis: debe poder captar la naturaleza de los números por medio de la inteligencia. La aritmética, pues, obliga al alma a poner en marcha el pensamiento para girarla hacia la contemplación de lo que es, para alcanzar la esencia (las alusiones a la necesidad de estudiar aritmética no se limitan a la República, sino que recorren la obra platónica: Chrm., 166a; Euthphr., 7b-c; Hp Ma., 303b-c; Grg., 451a y c, 453e; Tht,198a-c; Lg., 817d y 819b-c; Tht., 198b).

Si en la República Sócrates se burla del intento de Polemarco de encontrarle una justificación práctica al estudio de la matemática (R., 522c y sigs.), en el Filebo asegura que "si se apartaran de todas las ciencias las del número, medida y peso, lo 
que quedaría sería, por así decirlo, nulo" (Phlb., 55e). Y a continuación desdobla las ciencias en dos y anuncia que "hay dos aritméticas y dos metréticas y otras muchas que [...] tienen esa duplicidad bajo un único nombre (Phlb., 56c): la de la masa y la del filósofo, la actividad empírica y la actividad filosófica previa a la dialéctica, "el más verdadero conocimiento relativo al ser, a lo que realmente es y es siempre conforme a sí mismo por naturaleza" (Phlb., 58a).

Ante la pregunta de Sócrates acerca de los números en los cuales se halla la unidad tal como es considerada por los hombres asombrosos, Glaucón responde que en los números susceptibles de pensamiento, pero no de manipulación ( $R$, 526a). La importancia de la aritmética es tal que Sócrates propone que ya desde niños deben ser aprendidos tanto "los cálculos como la geometría y todos los demás estudios preliminares que deben enseñarse antes que la dialéctica" (R., 536d). Y lo mismo leemos en Leyes, 819c. Sócrates califica de "los más agraciados auxiliares" al medir, al contar y al pesar, pues ellos evitan que caigamos en el error de dejarnos gobernar por "lo que parece mayor y menor, más numeroso o más pesado" en lugar de por lo que "calcula, mide y pesa" (R., 602c-603a). Y tal es la función del alma racional, discernir según la medición y el cálculo, no al margen de ella, lo cual es propio de la parte inferior.

Esto nos conduce al pasaje en el cual Platón distingue una medida relativa y una medida absoluta como ejes del pensamiento matemático y dialéctico respectivamente (Polt, 283d y ss.). También a Teeteto le pregunta Sócrates en el diálogo homónimo cómo puede ser que confundamos el 11 con el 12 si "uno y otro son cosas que solamente nos representamos por medio del pensamiento" (Tht., 195e), a lo que aquel responde que solo podrá darse tal confusión si nos referimos a cosas que vemos o tocamos, nunca al 11 y al 12 del pensamiento. Sócrates concluye entonces que el 11 y el 12 o el 5 y el 7 en sí mismos "son los recuerdos impresos en la tablilla de cera respecto a los que no es posible tener opiniones falsas" (Tht., 196a). Ese recuerdo que está en el alma es el que la formación matemática debe recuperar.

En esta línea y para cerrar este apartado, me gustaría llamar la atención sobre un pasaje del Eutifrón en el que Sócrates orienta la discusión sobre la piedad hacia los asuntos que producen "enemistad e irritación en la disputa" (7b) y 
argumenta que son "lo justo y lo injusto, lo bello y lo feo, lo bueno y lo malo" (7d), pero no "lo mayor y lo menor" (7c), pues para eso bastaría con recurrir al cálculo. La importancia de este pasaje reside en que la ciencia, en este caso la rama de la matemática que conocemos como aritmética, prepara el camino que debe seguir la filosofía, que no es otro que alcanzar la idea de lo que cada cosa es para convivir en paz lejos de la enemistad y la disputa, pues "si no pudiéramos llegar a una decisión adecuada, nos haríamos enemigos" (7d). En definitiva, el conocimiento de lo inteligible como condición de posibilidad de una convivencia pacífica y un gobierno justo.

\section{Geometría}

En tanto "la geometría es el conocimiento de lo que siempre es" (R., 527b) o, en términos más actuales, "una lógica de los invariantes ocultos en toda formación” (BADIOU, 2007, p. 103), obliga a contemplar la esencia. En el Filebo, Sócrates se refiere a "la geometría que emplea la filosofía" (Phlb., 56e) y en la República nos invita a examinar si la geometría hace que el alma de los guardianes divise más fácilmente la Idea del Bien (

Si la geometría es el conocimiento de lo que existe eternamente, en lugar de decir "cuadrados", el geómetra debería decir que un cuadrado existe (eternamente); en lugar de decir que extiende un segmento recto, debería decir que el segmento es (eternamente) parte de un segmento más largo. Ninguno de los elementos necesarios para el conocimiento de un círculo enumerados en la Carta VII (nombre, definición, imagen, conocimiento en sí y objeto en sí, cognoscible y real) "le ocurre al círculo mismo al que se refieren todas las representaciones, pues es distinto a todas ellas" (Ep. VII, 342a-d). Dado que la geometría ideal trata exclusivamente con el dominio del ser eterno, un lenguaje geométrico que sugiera que el geómetra influye en el objeto que estudia, lo mueve o lo crea, es inexacto, impreciso o directamente erróneo (WEDBERG, 1955, p. 59). Platón se ríe de estos fingidores que hablan como si obraran, pues dicen cosas tales como "cuadrar”, “aplicar”, “añadir”. Los discursos de los geómetras se 
sirven de figuras visibles, no porque piensen en estas que dibujan, sino en aquellas cosas a las cuales estas se parecen, discurriendo en vista al Cuadrado en sí y a la Diagonal en sí. Los geómetras se sirven de estas imágenes para "divisar aquellas cosas en sí que no podrían divisar de otro modo que con el pensamiento ( $\delta \dot{\varepsilon} \alpha u ̛ \tau \alpha ̀$

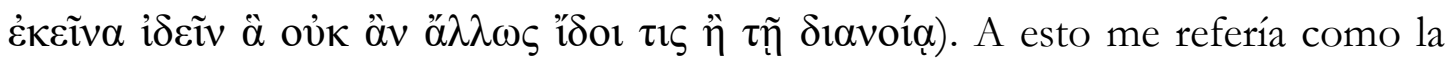

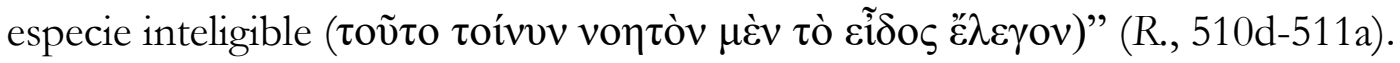

Platón es el primer autor en el que se encuentra referencia explícita al estilo matemático, a la manera de expresarse los geómetras, a su lenguaje, opuesto a la acción, al obrar y a la manipulación práctica propia de las cosas materiales (DE LORENZO, 1988, p. 52). Esto no quiere decir que Platón rechace que la matemática exija una acción para lo que necesite de la construcción, de la figura, único signo al que puede hacer referencia, puesto que el número aparece como una manipulación de los cálculos con guijarros en el gnómon. Uno de los ejemplos de la imperiosa necesidad de construcción gráfica es el célebre pasaje de Menón, 82-87, en el que Sócrates demuestra la duplicación del cuadrado construyendo materialmente sobre una figura.

Platón utiliza esta necesidad constructiva y manipuladora para introducir el concepto de reminiscencia. La matemática versa sobre objetos ideales, Formas puras que el hombre ha de captar por medio de la razón, no de los sentidos. El ejercicio mayéutico que Sócrates practica con el esclavo para resolver un problema geométrico favorece la anámnesis, el recuerdo de las ideas inmutables que el alma

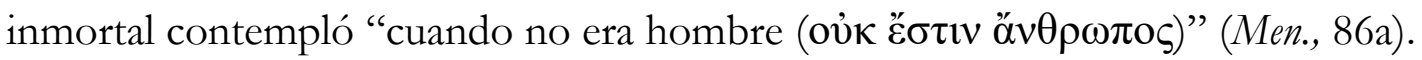

De ahí que la matemática, considerada en toda su pureza, sea independiente de cualquier construcción y de cualquier tipo de lenguaje material u oral. Pero la construcción gráfica y el empleo de signos especiales, imagen de esta Forma, son lo que posibilita que el hombre alcance la visión de la Forma pura mediante la reminiscencia (Phd., 72e; Phlb., 34c). En su trabajo de descubrimiento y exposición, el matemático procede hasta la Forma pura mediante construcción, mediante elevación desde lo sensible - la figura, el cálculo y la manera de disponerlos - y lo manual - la acción de cuadrar, de aplicar áreas - (DE LORENZO, 1988, p. 54). Los geómetras, los maestros del cálculo y los astrónomos se dedican a encontrar las figuras que ya existen, no a producirlas, y 
una vez que las han encontrado, como no saben qué hacer con ellas, se las entregan a los dialécticos (Euthd., 290b-c).

Que Platón opte por un ejemplo geométrico tan técnico en Menón, 82b$85 \mathrm{~b}$, para demostrar que todo aprendizaje es reminiscencia y debe ser interpretado es, según Vlastos, un intento de mostrar sus propios avances en el estudio de la matemática y avisar de algún modo a los lectores, oyentes o discípulos, de que, si no trabajan lo suficiente esta ciencia, no podrán ser capaces de seguirle y no podrán dedicarse a la filosofía (1988, p. 380). De ahí que Platón mandara inscribir en el frontispicio de la Academia: "No entre nadie que no sepa geometría"

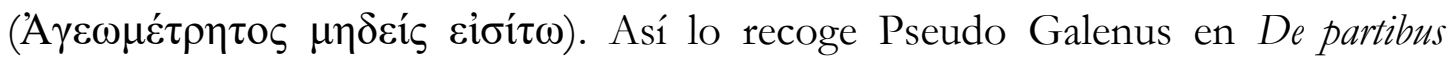
philosophiae, 2.5, anécdota que SOLANA DUESO (2008, p. 416) considera improbable, frente a una mayoría que no duda de su veracidad:

\footnotetext{
El valor de la matemática como propedéutica en la formación del filósofo y la concepción de los entes matemáticos como intermediarios entre el mundo de las ideas y el mundo de las cosas, justificarían la clásica frase que Platón habría estampado en el pórtico de la Academia, impidiendo su ingreso a los ignorantes en geometría (REY PASTOR y BABINI, 1997, p. 59).
}

Estos dos autores destacan la relación bidireccional entre el fomento del estudio de la matemática por parte de Platón y las aportaciones de los matemáticos que salieron de la Academia.

Al tratar con una jurisdicción del mundo ideal, la geometría es puesta en íntimo contacto con la ciencia de la dialéctica. Las últimas hipótesis de la geometría, las cuales el geómetra mismo debe asumir como garantía sin prueba, son probadas dentro de la dialéctica, y la fundación última de esa prueba es suplida por la Idea del Bien (WEDBERG, p. 59-60). Cuando Aristóteles en sus Analíticos posteriores insiste en la necesidad de que cada ciencia esté basada en premisas relativas al género concreto de esa ciencia, está rechazando implícitamente la propuesta platónica de fundamentar la matemática sobre una ciencia dialéctica general. Las últimas hipótesis de la filosofía geométrica deben encontrar su justificación dentro de la más alta de las ciencias, la dialéctica, el estudio filosófico general de las Ideas. En la filosofía geométrica la evidencia viene de arriba: los axiomas son, desde el punto de vista de la certidumbre, anteriores a los teoremas deducidos. 


\section{Estereonomía}

Platón se refiere a la estereonomía en dos de sus diálogos. Le asigna la segunda posición en la serie de las materias de estudio propias de los hombres libres, la medida de la extensión, la superficie y la profundidad, que hace referencia a la geometría y a la solidez de los cuerpos (en Lg., 817e). Y la describe como lo que concierne a la tercera dimensión, la dimensión de los cubos, "cuanto

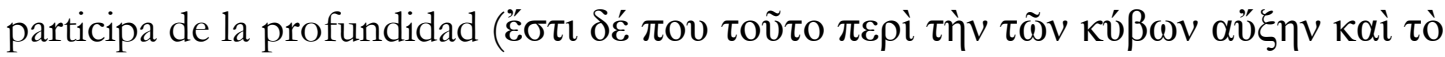

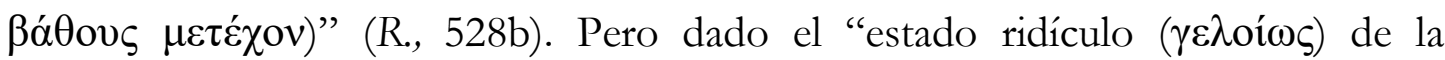
investigación" (R., 528d8-e1) en lo que a la estereonomía se refiere, enseguida es dejada de lado en beneficio de la astronomía. La relevancia de la estereonomía no se revela en la República, sino en el Timeo, donde se muestra su papel como llave del estudio de lo sensible, lo físico, lo tridimensional, particularmente a través de la generación simétrica de los poliedros (LANZA, 2015). Los cinco poliedros regulares convexos son, por tanto, uno de los objetos de esta ciencia. Como sugirió Jairo ESCOBAR MONCADA, toda la interpretación del Timeo debe pasar por "la relación entre espacio y elementos" (1995, p. 300).

\section{Astronomía}

La astronomía estudia el movimiento de los sólidos (R, 529d1-2), versa sobre "el curso de los astros, del sol y de la luna y sobre la relación de velocidades de unos con otros" (Grg., 451c), es decir, estudia "las revoluciones de los cuerpos celestes, cómo es el movimiento natural de unos en relación con los otros" (Lg., 817e). La astronomía es la tercera de las materias dictadas por el Ateniense en las Leyes. Tanto en este diálogo como en R. VII, describe Platón cómo la astronomía educa el pensamiento abstracto. Glaucón explica a Sócrates que "la astronomía obliga al alma a mirar hacia arriba y la conduce desde las cosas de aquí a las de allí en lo alto" (R., 529a), pero este replica que, tal y como está en ese momento la investigación filosófica, no ocurre de ese modo, sino justamente del contrario. Aunque los bordados visibles del cielo sean lo más bellos y perfectos que pueden 
ser, les falta mucho respecto a los verdaderos (R., 528d). Aquellos solo nos sirven como ejemplos (R., 529d7) para el estudio de estos. La propuesta de Sócrates es abandonar la dedicación a la astronomía desde los ojos y abordarla desde la parte divina del alma, la inteligencia: "nos serviremos de problemas en astronomía como lo hicimos en geometría, pero abandonaremos el cielo estrellado, si queremos tratar a la astronomía de modo de volver, de inútil, útil, lo que de inteligente hay por naturaleza en el alma" (R., 530c). Al igual que hay dos clases de aritmética, la filosófica y la popular, también hay dos clases de astronomía. VEGETTI ha destacado esta "deriva antiempirista" y se refiere a "una especie de astronomía ciega y una musicología sorda” (2012, p. 189).

En las últimas páginas de las Leyes, a colación de la formación de los miembros de la Junta Nocturna de magistrados, Platón establece las condiciones que debe cumplir un hombre para ser piadoso: primera, comprender que el alma es lo más antiguo de todo lo generado, que es inmortal y que gobierna todos los cuerpos; y segunda, captar el intelecto universal que se encuentra en los astros, contemplar la unión filosófica de estos y aplicarla a los hábitos del carácter y las leyes para “dar la definición de todo aquello que la tiene” (Lg., 967e4-968a1). Aquí se muestra que el estudio y la comprensión no quedan restringidos por Platón a lo puramente individual, sino que tienen su expresión en forma de ética y de política, de lo que tiene que ver con el éthos y con el nómos que regula la vida en la pólis. Con otras palabras, pero con el mismo sentido, escribirá posteriormente Aristóteles que no estudiamos para saber qué es la virtud, sino para ser mejores (EN., 1103b27-29). La brecha entre ciencia y religión abierta por Anaxágoras y los meteorólogos es salvada por Platón matematizando la astronomía. "La justificación última del mundo sensible es de carácter ético" (ELENA y ORDÓÑEZ, 1988, vol. I, p. 21). "No es solo un interés teórico el que tiene Platón por la ciencia de los astros. Su teoría posee, ante todo, implicaciones político-éticas" (LISI, 1991, p. 98). Platón investiga las causas de los fenómenos naturales por motivos éticos, porque los fenómenos naturales muestran un orden. Por eso la certeza de Timeo de que "sin la necesidad no es posible comprender la

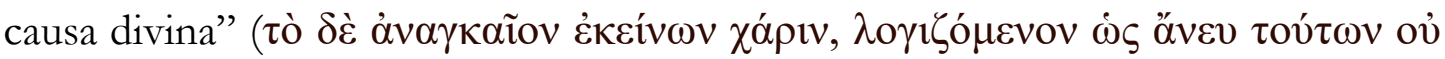




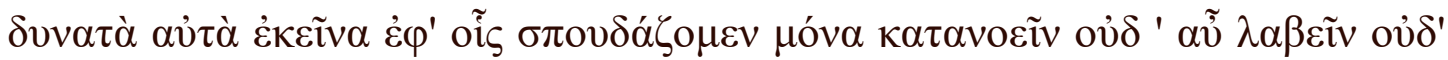
$\alpha \ddot{\alpha} \lambda \omega \varsigma \pi \omega \varsigma \mu \varepsilon \tau \alpha \sigma \chi \varepsilon i v)$ (Ti., 69a). La filosofía natural obedece a una motivación teológica y subraya la existencia de una causa final en el universo. La concepción platónica del orden cósmico está impregnada de alusiones éticas y sus modelos cosmológicos y su uso de la analogía microcosmos - macrocosmos tienen una significación moral. "Con Platón aparece el ideal de astronomía teórica que trasciende el mero registro de datos observacionales" (ELENA y ORDÓÑEZ, 1988, vol. I, p. 20).

\section{Música}

Por el mismo motivo que a Sócrates le interesa la astronomía que se aprehende con la inteligencia, y no con los ojos, se ríe de los músicos que anteponen a esta los oídos en lugar de examinar el movimiento armónico (R., 530d).

Arquitas "ofreció un principio, en forma de una teoría sobre las medias y las proporciones, evidentemente diseñado para la tarea que ha propuesto el Sócrates de Platón: la tarea de explicar por qué, desde un punto de vista matemático, ciertos grupos de relaciones están bien formados, matemáticamente coordinados o 'concordantes', mientras que otros no lo están" (BARKER, 1994, p. 133).

Los guardianes deberían ser educados no solo en gimnasia, sino también en música (R., 530d-531c; Ti., 18a). Al final de la descripción de las obras de los dioses menores, autores del cuerpo humano, en el pasaje dedicado a la voz y el oído, cuenta Timeo que la música y el ritmo son dones que las Musas regalaron a los hombres para que pusieran orden en la revolución inarmónica de su alma (Ti., 47c-e). Y ya al final del diálogo, Timeo destaca la importancia de la música para la salud: lo mismo que quien ejerce una actividad intelectual no debe olvidarse de hacer gimnasia para mover adecuadamente su cuerpo, quien se dedica a modelar este, si quiere ser llamado bello y bueno, tiene que complementar su ejercicio físico con el movimiento del alma que facilitan la música y la filosofía (Ti., 88c). También en la fabricación del alma del mundo introdujo el demiurgo proporciones matemáticas que se corresponden con la escala musical: su estructura comprende cuatro octavas, una quinta y un tono, pero esto no implica 
que Platón tenga ninguna intención de teorizar sobre la música de las esferas (GARCÍA CASTILLO, 1997, pp. 17-30).

La música es la última de las materias del curriculum matemático (R., 522c531d). Con ella, Platón da paso a la dialéctica con las siguientes palabras: "todo esto no es más que un preludio ( $\pi \rho 00 i ́ \mu 10 v)$ a la melodía que se debe aprender"

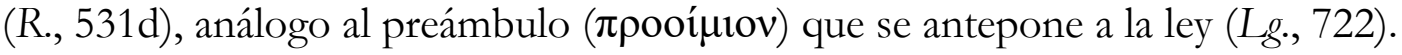

Desde el momento mítico en el que el demiurgo ordena lo existente en el Timeo asociando los elementos naturales a los poliedros regulares, la matemática hace posible el ascenso de lo humano a lo divino. En el Timeo, se nos dice formalmente que si las determinaciones numéricas y geométricas se introducen en la materia, es por la acción de la inteligencia. Así, mientras que el mecanismo moderno atribuye a la materia propiedades geométricas, en Platón, la geometría toda está del lado de la inteligencia (BROCHARD, 2008, p. 106).

Si bien los diálogos platónicos son uno de los principales fundamentos sobre el que se construirá la visión matemática del mundo que se impondrá de la Modernidad en adelante, la interpretación del Timeo en ocasiones ha extraído el lenguaje geométrico de su contexto, un mito cosmológico. Esta es, ahora sí, la verdadera cima, descubrir no que el mundo es matemático, sino por qué es matemático: por la previsión ( $\left.\pi \rho \operatorname{vov}_{\mathbf{v}} \alpha\right)$ divina (Ti., 44c), porque el demiurgo, que es inteligente, bueno sin envidia ni maldad, lo ha ordenado así (Ti., 30ab) gracias a los poliedros regulares y, una vez cumplida su tarea, se retira y deja en un mundo sin dioses en el que el único rastro de la divinidad es la estela que el dios deja en su retirada: la matemática.

\section{Dialéctica}

Es imposible entender la ontología griega sin referencia a su orientación específicamente matemática, solo la dialéctica puede abrir el reino del ser, solo ella


Uno y el Bien tal como son (KLEIN, 1992). 


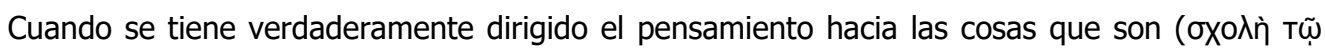

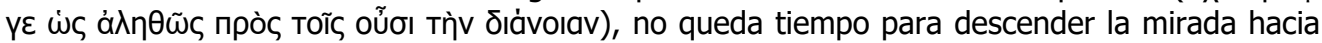
los asuntos humanos y ponerse en ellos a pelear, colmado de envidia y hostilidad; sino que, mirando y contemplando las cosas que están bien dispuestas y se comportan siempre del

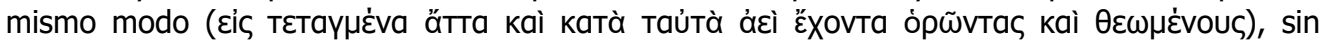

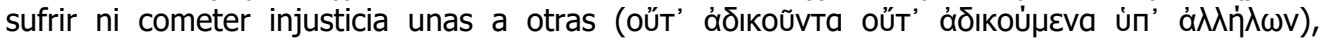

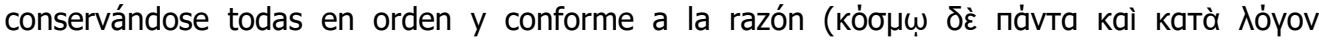

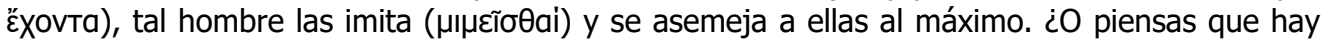

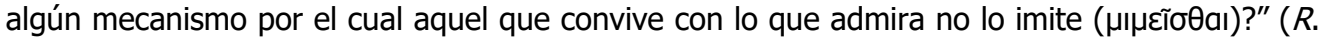
500c-d).

Tras exponer el curriculum matemático asegura Platón que "el poder dialéctico solo se revelará a aquel que sea experto en los estudios descritos" ( $R$. 533a). Solo quien se ha dedicado durante diez años de su vida a la matemática consigue liberarse de las pasiones y de las ataduras de los sentidos y girar la cabeza hacia la Idea del Bien (VLASTOS, 1988, p. 364). Y es que la geometría y demás ciencias afines solo "nos hacen ver lo que es como en sueños" (R. 533b), pero no nos sirven en la vigilia, porque se sirven de supuestos de los cuales no pueden dar cuenta y pretenden construir una ciencia conectando a algo desconocido los pasos intermedios del razonamiento y la conclusión. En ese sentido, sería más correcto no llamarlas ciencias, sino "pensamiento discursivo" (diánoia) (R. 533d), superior a la opinión (dóxa) e inferior a la ciencia (epistéme). En el corazón de la República, en la alegoría de la línea, (R. 509d-511e) Platón sitúa “el Cuadrado en sí y la Diagonal en sî́ (R. 510d-e) entre los objetos sensibles y las Ideas inmutables. El correlato epistemológico de esta ontología es que para liberarnos de las cadenas de la creencia (pístis), alcanzar la intelección (nóesis) y contemplar las Ideas, debemos primero pasar por el razonamiento discursivo (diánoia) gracias al que aprehendemos los objetos matemáticos. De ahí la necesidad de que los filósofosgobernantes estudien matemática y, por lo tanto, de que los guardianes deban superar el curriculum propuesto.

En el caso del intelecto, el conocimiento que almacena necesita coincidir con la «luminosidad» de las Ideas y del Bien para activarse y fortalecerse. En caso contrario, terminaría desapareciendo y hundiéndose en las opiniones sensibles, algo similar a lo que le sucede al alma del mundo cuando no es directamente dirigida por el demiurgo (cf. Plt. 273c4-e4) [...] el contacto directo es necesario para restablecer el orden. La unión del intelecto humano con la Idea del Bien produce la activación de la inteligencia, que, atesorada en el intelecto, se va 
desgastando paulatinamente, si no se renueva periódicamente ese contacto (LISI, 2018, p. 237).

Si bien la imagen platónica para ilustrar la relación entre el conocimiento y el alma es la de la tablilla de cera, una imagen actual podría ser la del conocimiento como material fotosensible, la cual ayuda a entender por qué lo bueno en sí tiene una doble dimensión ética y epistemológica, porque solo el Bien lo activa. Una vez que se entra en contacto con la Idea del Bien, ya se es capaz de gobernarse a sí mismo y ya se está en condiciones de gobernar a los demás, algo de lo que el tirano siempre será incapaz precisamente por su desmesura, diametralmente opuesta a la matemática.

La parte activa del alma ha sido para toda la ética griega la encargada de domesticar las pasiones y de dirigir el recto funcionamiento del compuesto anímico. Lejos de tratarse de una represión sin sentido, el logístico conduce prudentemente a la porción apetitiva del alma o de la ciudad de manera que se alcance la armonía general a partir del gobierno de sí [enkrateia] y, gracias al mismo, el correcto mandato sobre lo otro-de-la-razón (GARCÍA PONZO, 2010, p. 31).

La matemática es en Platón un entrenamiento del alma en el siguiente sentido. La matemática es capaz de sacar el alma del caudal del devenir para llevarla a lo que es (R, 521d3-4). Gracias a la ejercitación matemática, el guardián se convierte en dialéctico, lo que le permite contemplar las Ideas. Es entonces cuando, ante la visión de lo que es divino y ordenado, el dialéctico quiere convertirse él mismo en un ser divino y ordenado, en la medida de lo posible para

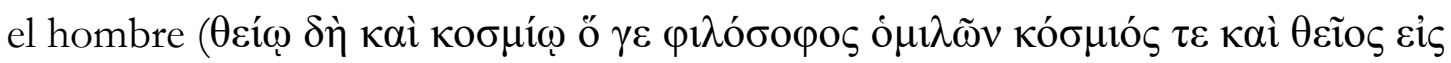

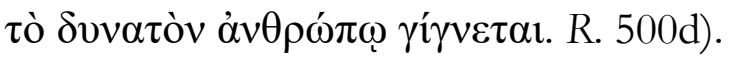

\section{El método matemático y el método dialéctico}

Cuando Platón enfoca la matemática como propedéutica de la filosofía, distingue el método hipotético-deductivo, razonamiento propio del geómetra, y el dialéctico, propio del filósofo, subordinando el primero al segundo (DE 
LORENZO, 1974, p. 43). Dado que el matemático no tiene la habilidad de sacar conclusiones probables y formar opiniones en argumentación metafísica, la matemática "no es más que el preludio de la tensión real que tenemos que aprender" (CAJORI, 1920, p. 58). Según Cherniss, la influencia de Platón en los discípulos de la Academia no fue la de un matemático técnico con la habilidad de hacer grandes descubrimientos por sí mismo, sino la de un crítico inteligente del método,

y fue por su crítica del método, por su formulación de los problemas más amplios a los que el matemático debería dirigirse, y por despertar el interés en las matemáticas en aquellos que se interesaron por la filosofía, que dio un gran impulso al desarrollo de la ciencia" (1945, p. 66).

Sin embargo, FOWLER destaca el nivel de los conocimientos matemáticos de Platón:

Si bien el principal interés de Platón estaba en la dialéctica, por lo que consideraba las matemáticas solo como una preliminar, no obstante muestra un conocimiento detallado de las características y problemas importantes de las matemáticas técnicas, y no hay indicios de que no pudiera comunicarse en igualdad de condiciones con los matemáticos que parecen haber dominado, si no integrado, el grupo de amigos y asociados que se reunieron a su alrededor (1999, p. 104).

A pesar de la proximidad que hay entre ambos métodos, el matemático y el filosófico (ver, por ejemplo, Euth., 290b), estos nunca podrán identificarse, porque la matemática tiende hacia la dialéctica sin alcanzarla jamás, “de hipótesis en hipótesis nunca toca lo absoluto” (REY, 1961, p. 212), como una asíntota tiende hacia su curva.

\begin{abstract}
Toda la ciencia verdadera, toda Matemática, es una persecución, una caza de la verdad, pero una persecución a ciegas. [...] La ciencia no puede por sí misma fundar sus hipótesis sobre un principio evidente por sí (índice en sí mismo de su propia verdad, índice inmutable, eterno y siempre presente). En consecuencia, es incapaz de dar cuenta plenamente de sí misma, de explicitar su inteligibilidad. Solo lo puede hacer la dialéctica (REY, 1961, p. 208).
\end{abstract}

Se comienza la búsqueda por el fin, conociendo únicamente el punto de partida, pues fue admitido, y el lugar donde se pretende llegar. Es decir, "no arranca desde el principio, sino desde el final, y atraviesa el discurso como un nadador que nadara de espaldas y hacia atrás" (Phdr, 264a). Para Platón, el método propiamente filosófico es el método dialéctico (he dialektiké méthodos). 
Cuando se intenta por la dialéctica llegar a lo que es en sí cada cosa, sin sensación alguna y por medio de la razón, y sin detenerse antes de captar con la inteligencia misma lo que es el Bien mismo, llega al término de lo inteligible como aquel prisionero al término de lo visible ( $R$. 532a).

El método dialéctico "es el único que marcha remontándose más allá de los supuestos, ascendiendo hasta el principio mismo" (R. 533c) y empuja al ojo

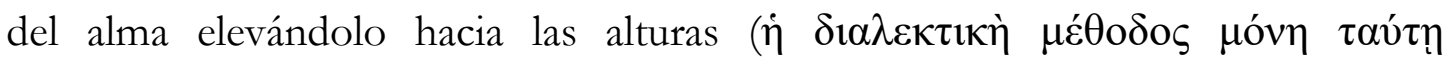

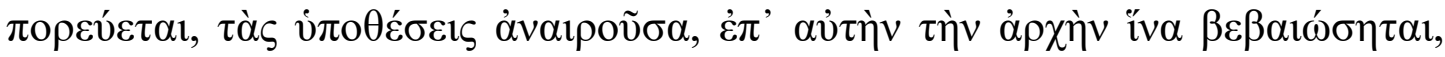

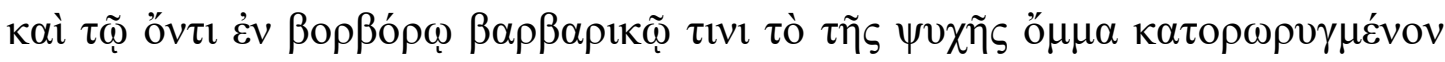

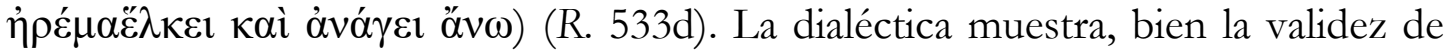
los supuestos, bien su insuficiencia por falta de fundamentación. La dialéctica "se pone en movimiento a partir de la discusión de las hipótesis para elevarse más allá de ellas hasta alcanzar un nivel ya no hipotético. Se trata de una comprensión/descripción de la idea en cuestión que parece ya irrefutable (VEGETTI, 2012, p. 204). O, si no irrefutable, sí "más difícil de refutar" (Phd, $85 c)$.

\footnotetext{
Solo la dialéctica puede abrir el reino del ser verdadero, puede dar lugar a los poderes de la dianoia y puede revelar el Ser, el Uno y el Bien tal como son más allá de todo tiempo y toda oposición, en sí mismos y en verdad (KLEIN, 1992, p. 79).
}

Únicamente los estudios dialécticos "tienen el mismo poder de elevar lo mejor que hay en el alma hasta la contemplación del mejor de todos los seres"

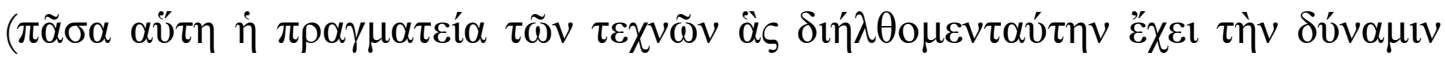

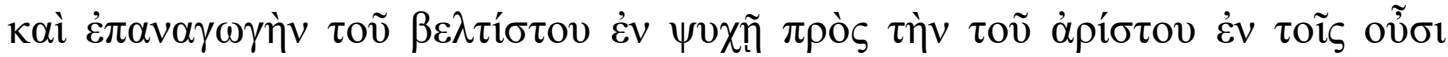

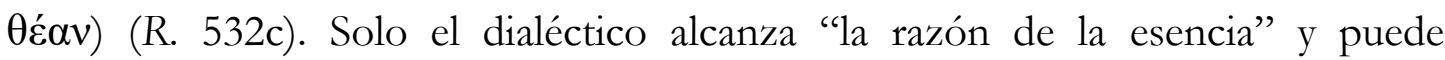
"distinguir la Idea del Bien con la razón, abstrayéndola de las demás" (R. 534b).

El método y el resultado de la dialéctica son "de naturaleza proposicional; la dialéctica actúa en el discurso, alcanza el discurso sobre la esencia y posteriormente debe dar cuenta de ello por medio del discurso (logos)" (VEGETTI, 2012, p. 205). La cuestión es si el carácter proposicional de este conocimiento da lugar a definiciones inmutables de entes noético-ideales o solo los delimita. 


\section{La influencia del desarrollo matemático en la concepción platónica de la dialéctica}

Según WEDBERG, "la filosofía de las matemáticas platónica” (1955, pp. 17, 20) está recogida en la República y en el Filebo y es la doctrina que Platón debe de haber enseñado cuando Aristóteles lo conoció. Lo poco que conocemos sobre las lecciones de Platón sobre el Bien confirma que el viejo Platón abrazó una filosofía matematizante que en algunos casos iba más allá de lo escrito por él mismo en sus diálogos. Esto es lo que Aristóteles llamó las "doctrinas no escritas" (Met. A 6 987a29-988a17), que dieron lugar a la interpretación de la escuela de Tübingen, encabezada por Konrad GAISER y Hans KRÄMER. Y también a la división que establece Leo STRAUSS entre una escritura exotérica, "popular y edificante" (1996, p. 90) y otra esotérica, orientada a quienes "aman pensar" (1996, p. 77).

NOVAK defiende que el desarrollo del estudio de los números inconmensurables, como raíz de dos y raíz de tres, influyó en la filosofía platónica, que los tomó "como un modelo que influyó su dialéctica final, colección y división" (1982, p. 72). La argumentación de NOVAK procede contrastando los últimos diálogos con los de juventud, en los que se buscan definiciones, las cuales no pueden ser formuladas a causa del Ě̉ $\lambda$ $\gamma \chi 0 \varsigma$ socrático, de ahí el carácter aporético de estos. En los diálogos medios Platón abandona este método que no alcanza ninguna definición concluyente en favor de la presentación definitiva de sus doctrinas éticas y políticas.

\footnotetext{
Los desarrollos en geometría y aritmética griegas que tenían lugar en ese momento en la Academia tuvieron un impacto significativo en la transición desde la teoría media a la tardía, así como la aritmética pitagórica heredada afectó a la transición de los diálogos tempranos a los medios" (NOVAK, 1982, p. 78).
}

La hipótesis de NOVAK es que el método dialéctico de los últimos diálogos responde al interés en el trabajo matemático sobre cantidades inconmensurables y su impacto sobre la definición. Las formas, que anteriormente se habían modelado en unidades, ahora se modelan en cantidades 
continuas y la comprensión intuitiva clara de una Forma es reemplazada por la articulación proposicional compleja en una definición. "Las formas se definen a través de una koinonia como los inconmensurables se definen en un continuo. La definición de cualquier forma dada nunca es exhaustiva, así como la aproximación de una cantidad inconmensurable nunca termina" (NOVAK, 1983, p. 24).

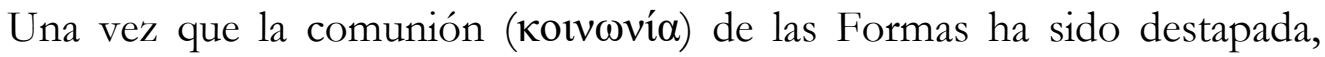
según el Extranjero, se hace necesaria, consecuentemente, la ciencia capaz de enfrentarse a este entramado, la dialéctica. Las Formas son esencialmente relaciones y "el lugar primordial de relacionarse las Formas entre sí es el juicio predicativo” (MONTSERRAT, 1995, p. 144). La relación de lo uno y lo múltiple tiene lugar primordialmente en el juicio o enunciado. A través del enunciado, todo individuo y toda idea son introducidos en los sistemas conceptuales, dentro de los cuales son definibles y cognoscibles. La filosofía de Platón rompe con la ontología de Parménides y establece la multiplicidad de las Formas y su carácter compuesto y analizable. Lo idéntico es improductivo en relación consigo mismo, por lo que es relacionado con lo diferente. Los conceptos trascendentales de identidad y diferencia presuponen los conceptos correlativos de lo uno y lo múltiple. Las Formas no son entidades simples, sino compuestas y analizables. Identidad y diferencia son conceptos correlativos. La nueva teoría de las Formas implica que cuatro de las ocho hipótesis del Parménides ponen lo uno y lo múltiple en relación mutua. Con las ocho, Platón puso de manifiesto que la eliminación del carácter relacional de una Idea conduce a la absoluta imposibilidad de su conocimiento (MONTSERRAT, 1995).

Platón recurre al ejemplo del arte de tejer para explicar qué es un modelo y para referirse a la política y a la trabazón de las Formas entre sí. Este ejemplo emparenta el Sofista con la Lisístrata de Aristófanes, cuando esta se encara con el magistrado y le espeta: "Si tuvieses una pizca de sentido común, seguiríais en política el ejemplo que os damos con nuestras lanas” (vv. 572-573).

Esta evolución de la ontología platónica podría tener su correlato político en la evolución que se da entre la República y las Leyes, pues si bien en la primera ya se insiste en la necesaria comunidad de placer y dolor, es en la segunda donde se 
especifica cuáles son los elementos concretos que favorecen la cohesión social, como los coros, las danzas y el vino.

\title{
El camino de descenso: la vuelta a la caverna. El gobierno justo de la pólis.
}

"Después de eso debes hacerlos descender nuevamente a la caverna y obligarlos a mandar en lo tocante a la guerra y a desempeñar cuantos cargos convienen a los jóvenes, para que tampoco en experiencia queden atrás de los demás” (R. 539e). En palabras de EGGERS LAN: “para qué debe llegar el filósofo hasta la cima. No es para quedarse en ella, sino para saber lo que debe hacer en el Estado: aquí Platón, una vez más, es filósofo político” (2000, p. 7). Es lo que VEGETTI llamó "la katabasis ético-política de la filosofía en la caverna" (2012, p. 206). LISI va incluso más allá: “Como los héroes, el filósofo realiza una

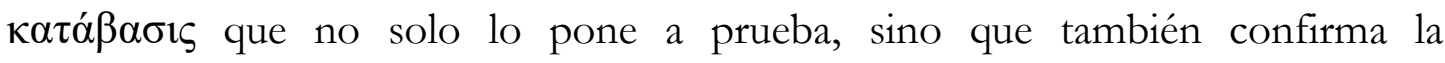
transformación ontológica que ha producido en él la Idea del Bien” (LISI, 2018, p. 249). La importancia no reside, entonces, en el proceso matemático, ni siquiera en la transformación ontológica del filósofo, sino en que este acontecimiento abre la posibilidad de un gobierno justo.

\begin{abstract}
La bondad que produce el contacto con el Bien impulsa a los guardianes a bajar al gobierno de la ciudad para ordenarla, como sucede al Demiurgo con la creación del mundo (Ti., 29e1-2), y en función también de su propio interés que consiste en el mantenimiento del orden social. La felicidad radica en la actualización de la virtud y no en el disfrute del placer en el sentido habitual del término. Por ello, el ejercicio de la política es fundamental para la realización de la felicidad filosófica (LISI, 2018, p. 248).
\end{abstract}

El filósofo será feliz si y solo si gobierna. El espíritu del filósofogobernante requiere una exhaustiva formación en las ciencias del quadrivium pitagórico como base preliminar ineludible del supremo conocimiento dialéctico del Bien, verdadera finalidad de los estudios filosóficos, de modo que, en toda actividad intelectual de la Academia, la matemática y, en especial, la geometría, alcanza una significación filosófica y un valor ético y político. El dialéctico es el único capaz, por un lado, de criticar la falta de cimientos de la política anclada en 
las sombras del poder, la riqueza y la gloria y, por el otro, de asegurar la verdad de los valores que deben constituir la norma, de "explicar el poder de la verdad para la vida moral y política de los hombres" (VEGETTI, 2012, pp. 206-207) y, por tanto, de gobernar la vida de los demás. Platón ha mostrado cómo las ciencias son útiles para alcanzar la felicidad en común, tanto porque orientan el alma hacia la verdad cuanto porque son "invertibles en conocimientos técnicos necesarios para la ciudad, desde la arquitectura a la estrategia” (VEGETTI, 2012, p. 207).

La descripción del estado de armonía musical entre cuerpo-alma como el estado más kata physin posible se relaciona, por otra parte, con el requerimiento del filósofo como gobernante óptimo, capaz de dotar a la ciudad de la mejor politeia posible a través de una operación de armonización en las potencias de la polis semejante a la realizada en su cuerpo y su alma, mediante un proceso de regulación de dichas potencias devolviéndolas a su estado natural. Leído este pasaje en el marco de la configuración del cosmos que establece Platón en el Timeo, la figura del político adquiere ciertos rasgos demiúrgicos. En tanto que configurador de un orden, su tarea discurre en paralelo a la de la divinidad: la armonización de principios opuestos entre sí para lograr un compuesto estabilizado (CANO CUENCA, 2018, p. 10).

A pesar de los defensores de la interpretación de la divinización del filósofo, si bien la matemática es condición de posibilidad de un gobierno racional, no garantiza un gobierno justo para siempre (Lg., 875b), sino que este deberá alejarse cíclicamente del poder. Sin embargo, el gobierno racional es más justo que el irracional, puesto que lo justo es que cada parte haga lo que le corresponde por naturaleza (R. 433b) y lo propio del alma racional es gobernar.

\section{¿Quién es el filósofo-gobernante en el siglo XXI?}

Para finalizar, regresemos a la actualización del platonismo llevada a cabo por Badiou, quien afirma que "La justicia es el nombre filosófico de la verdad en el campo político (2014, p. 42). Para él, la matemática es el mejor paradigma de la justicia en este sentido: en ella tenemos una libertad primitiva para elegir los axiomas, seguida de la determinación de las reglas lógicas, que nos obligan a aceptar las consecuencias de aquella elección primera. En nuestras propias palabras: el momento en el que Sócrates decide que es mejor sufrir injusticia que cometerla, determina que renuncie a escapar de la prisión a pesar de la ayuda de Critón y que tome la cicuta en el Fedón. 
Según la interpretación de Badiou, el gobernante justo sería el único ciudadano de la pólis que dejaría de ser un mero individuo y entraría en contacto con lo absoluto a través de una verdad, que posee un valor absoluto, universal e independiente. ("Individuo" es el animal humano que nace y muere y "sujeto" es aquello a lo que se incorpora el individuo cuando toca lo universal).

Debido a que en las actuales democracias no tiene sentido hablar del filósofo-rey, sino de que todos los que gobiernan o todos los que se gobiernan devengan filósofos (BADIOU, 2013), para que todos ejerzamos la democracia, es necesario universalizar los procedimientos de verdad (el matema, el poema, la política emancipatoria y la experiencia amorosa de contemplar el mundo desde la perspectiva del Dos).

El intelectualismo platónico restringía las doctrinas no escritas a la transmisión esotérica y la formación matemática a los filósofos gobernantes, pero en la actualización del pensamiento de Platón que hace Badiou el punto más revolucionario es que hace exclamar a Sócrates que todos seamos filósofos, que es lo mismo que abrir a todos el estudio de la matemática para que todos tengamos la posibilidad de asimilarnos al sujeto colectivo, entremos en contacto con lo absoluto real y gobernemos.

\section{Conclusiones}

1. Platón es el primer filósofo occidental en ocuparse del estatuto de los entes matemáticos y su proceso de conocimiento, del estilo matemático y del papel de puente que cumple la matemática entre pares de opuestos aparentemente irreconciliables: lo sensible y lo inteligible, lo cambiante y lo inmutable, lo humano y lo divino.

2. El convencimiento de la importancia y la necesidad de la formación y la ejercitación matemática condujo a Platón a establecer un modelo de curriculum cimentado en la aritmética, la geometría, la estereonomía, la astronomía y la música, dirigido a educar a los guardianes de los que debía salir el futuro gobernante. 
3. El curriculum del guardián-filósofo culmina en el estudio supremo: la dialéctica. Aunque la matemática es propedéutica de la filosofía y hay una continuidad entre la matemática y la dialéctica, los métodos de ambas son distintos; solo el dialéctico es el propiamente filosófico. La matemática permite al filósofo ascender hacia lo inteligible, ámbito en el cual necesita la dialéctica.

4. La convivencia con lo divino y ordenado es lo que, por imitación, hace que el guardián quiera volverse a su vez divino y ordenado y, por tanto, lo que convierte al dialéctico en alguien apto para gobernar. Es en este sentido que se afirma que estudiar matemática ayuda a dominar las pasiones.

5. La relación de Platón con la matemática es bidireccional: en un sentido, la fundación de la Academia supuso un impulso de los estudios matemáticos y su engarce con la política, mientras que en el sentido opuesto, la concepción platónica de la dialéctica pudo haber sido influida por el desarrollo del estudio de los inconmensurables. Esto se refleja en el paso de una ontología constituida por

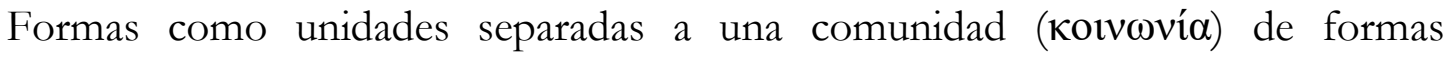
entrelazadas y combinadas entre sí ( $\left.\sigma v \mu \pi \lambda{ }_{0} \eta^{\prime}\right)$.

6. Esclarecer el papel de la matemática en el pensamiento platónico permite ver más claramente la dimensión política del conocimiento y entender la relación saber-poder en el pensamiento platónico, que suelda la filosofía a la política. El filósofo, una vez efectuado el ascenso desde el ámbito sensible hasta el inteligible, debe responder a la llamada de la pólis enferma, siempre en el caso de que esta muestre una buena disposición y lo reclame haciéndole girarse y dejar sus asuntos personales para descender de nuevo a la caverna y gobernar de forma justa a los prisioneros embrujados por un teatro de sombras.

7. En el platonismo de lo múltiple de Badiou, la formación matemática del filósofo-gobernante es ahora el matema, uno de los cuatro procedimientos de verdad a través de los cuales cualquier individuo tiene la posibilidad de abandonar sus condiciones particulares y asimilarse al sujeto universal. La actualización de Platón se vuelve, así, una crítica a Platón. 


\section{Referencias}

ARISTÓTELES. Ética Nicomáquea. Madrid: Gredos, 1985.

ARISTÓTELES. Fisica. Madrid: Gredos, 1998.

ARISTÓTELES. Metafísica. Madrid: Gredos, 1994.

ARISTÓTELES. Tratados de lógica 2. Organon. Madrid: Gredos, 1988.

BADIOU, A. Justicia, filosofía y literatura. Rosario: Homo sapiens, 2007.

BADIOU, A. La República de Platón. Buenos Aires: Fondo de Cultura Económica, 2013.

BADIOU, A. Filosofía y política: una relación enigmática. Buenos Aires: Amorrortu. 2014.

BARKER, A. Ptolemy's Pythagoreans, Archytas, and Plato's Conception of Mathematics. Phronesis, v. 39, n. 2, p. 113-135, 1994.

BROCHARD, V. Estudios sobre Sócrates y Platón. Buenos Aires: Losada, 2008.

CAJORI, F. Greek philosophers on the disciplinary value of mathematics. The Mathematics Teacher 13, n. 2, p. 57-62, 1920.

CANO CUENCA, J. Una educación política: apuntes sobre la función de la geometría en el Teeteto y la República, 2018. [Inédito]

CHERNISS, H. The Riddle of the Early Academy. Berkeley: University of California Press, 1945.

DE LORENZO, J. de. La filosofía de la matemática de Poincaré. Madrid: Tecnos, 1974.

DE LORENZO, J. de. Introducción al estilo matemático. Madrid: Tecnos, 1988.

EGGERS LAN, C. El sol, la linea y la caverna. Buenos Aires: Colihue, 2000.

ELENA, A.; ORDÓÑEZ, J. Historia de la ciencia. 2 vols. Madrid: UAM, 1988.

ESCOBAR MONCADA, J. Consideraciones generales sobre el Timeo de Platón. Estudios de Filosofía, n. 11, p. 297-304, 1995.

FOWLER, D. H. The Mathematics of Plato's Academy: A New Reconstruction. Oxford: Clarendon Press, 1999. (especialmente cap. 4. Plato's Mathematics curriculum in Republic VII, pp. 103-151)

GARCÍA CASTILLO, P. La música la educación en Platón. Música y educación: Revista trimestral de pedagogía musical, n. 31, p. 17-30, 1997.

GARCÍA PONZO, L. Un filósofo, otra vez. In: Badiou, A. La filosofía, otra vez: Madrid: Errata Naturae, 2010.

KLEIN, J. Greek mathematical thought and the origin of algebra. New York: Dover, 1992. 
LANZA, H. Matemática y física en el Timeo de Platón. Poliedros regulares y elementos naturales, Praxis filosófica. Nueva serie, n. 40, p. 895-112, 2015.

LISI, F. L. Astrología, astronomía y filosofía de los principios en Platón. Habis, n. 22, p. 97-112, 1991.

LISI, F. L. República VII 517a8-521c1. Emerita, n. 86, p. 233-252, 2018.

MONTSERRAT, J. Platón. De la perplejidad al sistema. Barcelona, Ariel, 1995.

NOVAK, J. Plato and the irrationals. Apeiron, v. 16, n. 2, p. 71-85, 1982.

NOVAK, J. Plato and the irrationals. Apeiron, v. 17, n. 1, p. 14-27, 1983.

PLATÓN. Diálogos. I. Apología. Critón. Eutifrón. Ion. Lisis. Cármides. Hipias menor. Hipias mayor. Laques. Protágoras. Madrid: Gredos, 1981.

PLATÓN. Diálogos. II. Gorgias. Menéxeno. Eutidemo. Menón. Crátilo. Madrid: Gredos, 1983.

PLATÓN. Diálogos. III. Fedón. Banquete. Fedro. Madrid: Gredos, 1986.

PLATÓN. Diálogos. IV. República. Madrid: Gredos, 1986.

PLATÓN. Diálogos. V. Parménides. Teeteto. Sofista. Político. Madrid: Gredos, 1988.

PLATÓN. Diálogos. VI. Timeo. Filebo. Critas. Madrid: Gredos, 1992.

PLATÓN. Diálogos. VII. Dudosos, apócrifos y cartas. Madrid: Gredos, 1992.

PLATÓN. Diálogos. VIII. Leyes (libros I-VI). Madrid: Gredos, 1999.

PLATÓN. Diálogos. IX. Leyes (libros VII-XII). Madrid: Gredos, 1999.

PLATÓN. Timeo. Buenos Aires: Colihue, 1999.

PLATÓN. Timeo. Santiago de Chile: Univ. Católica de Chile, 2003.

PLATÓN. Ión. Timeo. Critias. Madrid: Alianza, 2004.

PLATÓN. Timeo. Madrid: Abada, 2010.

REY, A. La madurez del pensamiento cientifico en Grecia. México: UTEHA, 1961.

REY PASTOR; J. BABINI, J. Historia de la matemática, I. Barcelona: Gedisa, 1997.

SOLANA DUESO, J. Los filósofos griegos y sus escuelas, Arbor. Ciencia y pensamiento y cultura, v. 184, n. 731, p. 413-422, 2008.

STRAUSS, L. Persecución y arte de escribir y otros ensayos de filosofía política. Valencia: Alfons el Magnànim, 1996.

VEGETTI, M. Platón. Madrid: Gredos, 2012. 
VLASTOS, G. Elenchus and Mathematics: a turning-point in Plato's philosophical development. The American Journal of Philology, v. 109, n. 3, p. 362-96, 1988.

WEDBERG, A. Plato's Philosophy of Mathematics. Estocolmo: Almquist \& Wiksell, 1955.

RECIBIDO: $18 / 11 / 2018$

APROBADO: 26/02/2020

RECEIVED: $11 / 18 / 2018$

APPROVED: 02/26/2020 